\title{
L'étude thermodynamique des mélanges liquides chlorure d'argent - chlorure de zinc et bromure d'argent - bromure de zinc à partir des diagrammes d'équilibres des phases
}

\author{
A. Wojakowska ${ }^{1}$, A. Górniak ${ }^{1}$ et A. Wojakowski ${ }^{2}$ \\ ${ }^{1}$ Laboratoire d'Analyse Thermique, Département de Chimie Minérale, Académie de \\ Médecine, 38 rue Szewska, 50139 Wrocław, Pologne \\ 2 Institut des Basses Températures et des Recherches Structurales, l'Académie \\ Polonaise des Sciences, BP. 1410, 50950 Wrocław, Pologne
}

\begin{abstract}
Résumé : Les diagrammes d'équilibre des phases des systèmes $\mathrm{AgCl}-\mathrm{ZnCl}_{2}$ et $\mathrm{AgBr}-\mathrm{ZnBr}_{2}$ sont obtenus par calorimétrie différentielle à balayage. Ils sont du type eutectique. Les propriétés thermodynamiques des mélanges liquides sont déterminées à partir des courbes de liquidus. Les résultats sont discutés en liaison avec la structure des sels fondus.
\end{abstract}

\section{INTRODUCTION}

Les chlorure et bromure de zinc ont la tendance de solidifier sous forme de verre. Pour cette raison les systèmes comportant les halogénures de zinc suscitent un intérêt en vue de l'élaboration de matériaux vitreux [1-3]. L'évolution des propriétés thermodynamiques en fonction de la composition et de la température pour le système binaire peut être utile pour une exploitation ultérieure du système à la recherche des matériaux vitreux dans les systèmes à plus de deux constituants.

Le diagramme d'équilibre des phases du système $\mathrm{AgCl}-\mathrm{ZnCl}_{2}$ a fait l'objet d'une seule étude [4]. La courbe de liquidus, reportée par Glistenko et Artemowa, a été obtenue par la méthode "visuelle", c'est-àdire par l'observation de l'apparition des premiers cristaux au refroidissement du mélange. Le diagramme d'équilibre des phases du système $\mathrm{AgBr}-\mathrm{ZnBr}_{2}$, semble-t-il, n'a pas été publié. L'étude de ces deux systèmes a été entreprise pour préciser les diagrammes d'équilibre des phases et calculer les variations de l'enthalpie libre de mélange à l'état liquide le long du liquidus.

\section{PARTIE EXPERIMENTALE}

Les chlorure et bromure d'argent sont préparés par la réaction de précipitation en solution aqueuse. Les précipités sont separés du liquide, chauffés et ensuite fondus. Toutes les expériences ont été effectués à la lumière rouge. Les halogénures de zinc sont des produits commerciaux de Aldrich Chem. Co. Les puretés de $\mathrm{ZnCl}_{2}$ et $\mathrm{ZnBr}_{2}$ sont respectivement $99.99 \%$ et $99.999 \%$.

Les mélanges $\mathrm{AgCl}-\mathrm{ZnCl}_{2}$ et $\mathrm{AgBr}-\mathrm{ZnBr}_{2}$ sont obtenus par synthèse directe dans des cellules en quartz scellées sous vide, en utilisant des sels de haute pureté, pesés à l'interieur d'une boîte à gants MBraun Labmaster 130, remplie de hélium. Les cellules sont ensuite utilisées dans des expériences de calorimétrie différentielle à balayage (DSC25 Mettler Toledo, logiciel STARe 6.0). L'étalonnage en température de cet appareil a été obtenu en étudiant la fusion des metaux de haute pureté (indium, étain, plomb, zinc). Les deux cellules, expérimentale et témoin, ont pour dimensions: diamètre externe $6 \mathrm{~mm}$ et hauteur environ $14 \mathrm{~mm}$. La surface du fond a été rodée afin d'être en bon contact thermique avec le capteur. Les expériences ont été realisées avec les vitesses de chauffe ou de refroidissement comprises entre 0.5 et $5 \mathrm{~K} \mathrm{~min}^{-1}$ sur le domaine de température $325-723 \mathrm{~K}$. 


\section{RESULTATS}

Nous avons réexaminé le système $\mathrm{AgCl}-\mathrm{ZnCl}_{2}$ et nous avons établi le système $\mathrm{AgBr}-\mathrm{ZnBr} 2$. Les deux diagrammes d'équilibre des phases sont de type eutectique. Sur tout le domaine de compositions on observe par DSC un pic d'eutexie à $503.5 \mathrm{~K}$ dans le binaire $\mathrm{AgCl}-\mathrm{ZnCl}_{2}$ et à $551.0 \mathrm{~K}$ dans $\mathrm{AgBr}-\mathrm{ZnBr}_{2}$. Pour déterminer les compositions des points eutectiques et confirmer l'absence des solutions solides, nous avons établi les diagrammes de Tamman relatifs aux invariants de l'eutexie. On obtient ainsi la fraction molaire de $\mathrm{AgCl}$ égale à 0.535 et celle de $\mathrm{AgBr}$ égale à 0.594 . Les points représentatifs de phases solides correspondent aux composants purs, indiquant que les domaines de solubilité mutuelle sont négligeables.

Etant donné que la miscibilité a l'état solide est très faible ou nulle, il a été possible de calculer les changements des grandeurs thermodynamiques relatives à l'état liquide en fonction de la fraction molaire: les enthalpies libres molaires partielles de mélange des composants $\Delta g_{1}$ (Figure 1) et les enthalpies libres molaires partielles d'excès des composants $\Delta g_{i}^{E}$ (Figure 2) ainsi que les enthalpies libres molaires intégrales et les enthalpies libres molaires intégrales d'excès pour les mélanges correspondants.

Ces grandeurs thermodynamiques sont obtenues à partir des relations classiques. Le calcul des enthalpies libres molaires partielles de mélange du composant qui cristallise à la température $T$ en équilibre avec la solution demande la connaissance des valeurs de l'enthalpie de fusion $\left(\Delta_{\text {fus }} H\right)$ et celle de la différence des chaleurs spécifiques de ce composant entre l'état liquide et l'état solide $\left(\Delta \mathrm{Cp}=\mathrm{Cp}^{\mathrm{l}}-\mathrm{Cp}^{\mathrm{S}}\right)$. Les valeurs utilisées [5-8] sont indiquées dans le tableau 1. Les enthalpies libres molaires partielles de mélange de l'autre composant sont calculées par intégration de l'équation de Gibbs-Duhem.

Tableau 1. Valeurs de $\Delta_{\text {fus }} \mathrm{H}$ et $\mathrm{Cp}=\mathrm{A}+\mathrm{B} \mathrm{T}+\mathrm{C} \mathrm{T}^{-2}$

\begin{tabular}{|l|l|l|l|l|l|l|l|}
\hline & $\begin{array}{l}\Delta_{\text {fus }} \mathrm{H} \\
\mathrm{kJ} \mathrm{mol}^{-1}\end{array}$ & Ref. & Phase & A & B & C & Ref. \\
\hline $\mathrm{AgCl}$ & 13.22 & 5 & $\begin{array}{l}\text { Liquide } \\
\text { Solide }\end{array}$ & $\begin{array}{l}57.3 \\
41.30\end{array}$ & 0.03323 & & 5 \\
\hline $\mathrm{AgBr}$ & 9.14 & 6 & $\begin{array}{l}\text { Liquide } \\
\text { Solide }\end{array}$ & $\begin{array}{l}67.722 \\
-63.839\end{array}$ & 0.21402 & 5896762 & 7 \\
\hline $\mathrm{ZnCl}_{2}$ & 16.1 & 8 & $\begin{array}{l}\text { Liquide } \\
\text { Solide }\end{array}$ & $\begin{array}{l}100.83 \\
59.83\end{array}$ & 0.03766 & & 8 \\
\hline $\mathrm{ZnBr}_{2}$ & 15.6 & 8 & $\begin{array}{l}\text { Liquide } \\
\text { Solide }\end{array}$ & $\begin{array}{l}113.8 \\
52.72\end{array}$ & 0.04351 & & 8 \\
\hline
\end{tabular}

\section{DISCUSSION}

Nos résultats montrent que les écarts à l'idéalité ne sont pas importants. Le système de bromures indique toujours des déviations positives, alors que le système de chlorures ne montre des déviations positives que dans la zone des compositions plus riches en chlorure de zinc, par contre dans la zone des compositions plus riches en chlorure d'argent apparaissent des déviations négatives.

Dans les mélanges $\mathrm{AgBr}-\mathrm{ZnBr}_{2}$ l'écart de l'enthalpie libre molaire intégrale d'excès de mélange ne dépasse pas $+800 \mathrm{~J} \mathrm{~mol}^{-1}$. Le maximum est voisin de $40 \% \mathrm{~mol} \mathrm{AgBr}$. L'effet d'asymétrie est alors nettement marqué. Dans les mélanges $\mathrm{AgCl}-\mathrm{ZnCl}_{2}$ l'écart de l'enthalpie libre molaire intégrale d'excès de mélange se situe entre $+380 \mathrm{~J} \mathrm{~mol}^{-1}$ (pour environ $20 \% \mathrm{~mol} \mathrm{AgCl}$ ) et $-625 \mathrm{~J} \mathrm{~mol}^{-1}$ (pour environ $70 \% \mathrm{~mol}$ $\mathrm{AgCl}$ ). Les déviations négatives dans les mélanges $\mathrm{AgCl}-\mathrm{ZnCl}_{2}$ permettent de prévoir des interactions de mélange plus importantes et impliquent une formation des complexes plus stables que dans les mélanges $\mathrm{AgBr}-\mathrm{ZnBr}_{2}$. 

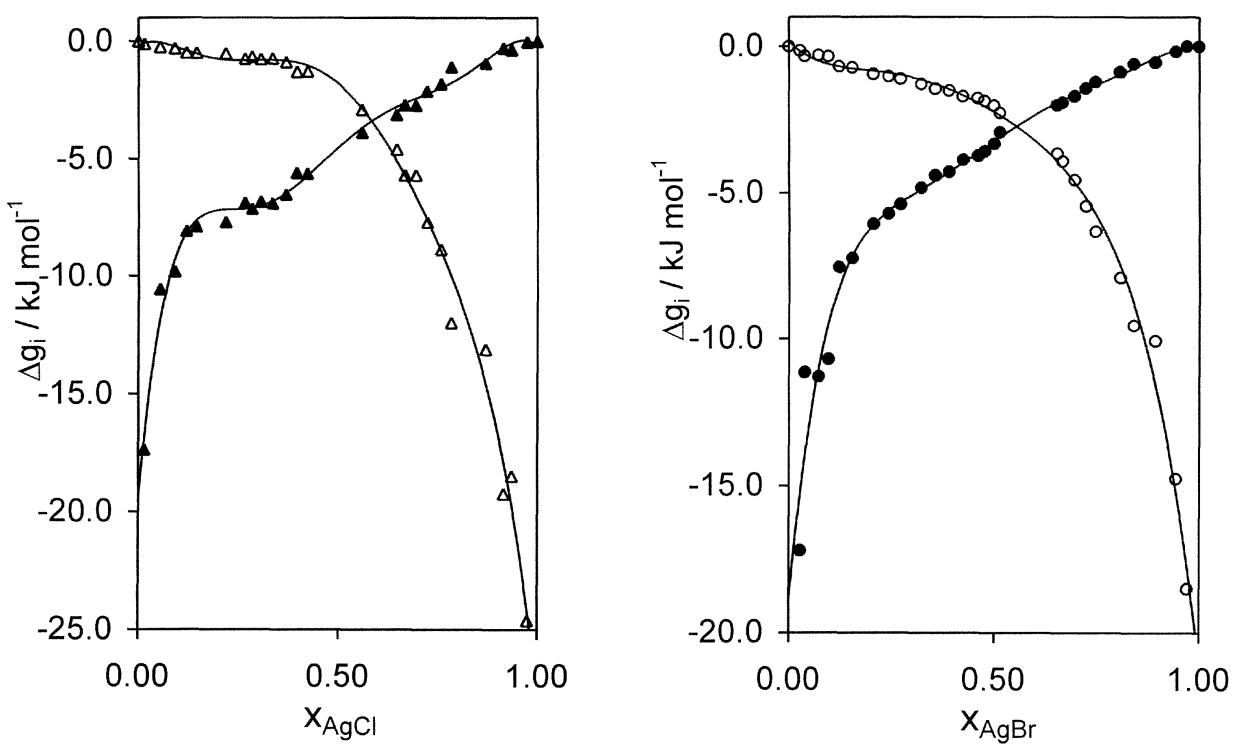

Figure 1: Enthalpies libres molaires partielles de mélange des composants $\Delta \mathrm{g}_{\mathrm{i}}$ pour le système $\mathrm{AgCl}-\mathrm{ZnCl}_{2}$ et pour le système $\mathrm{AgBr}-\mathrm{ZnBr}_{2}$
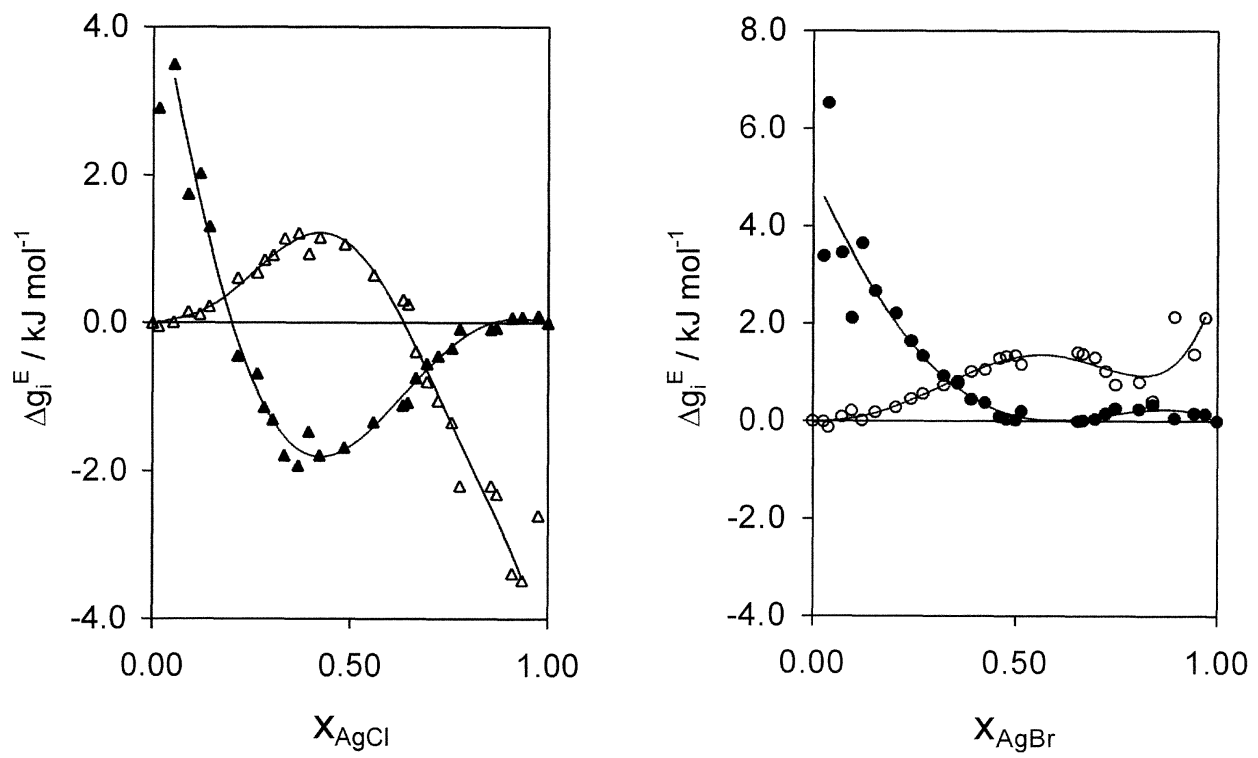

Figure 2: Enthalpies libres molaires partielles d'excès des composants $\Delta \mathrm{g}_{\mathrm{i}}^{\mathrm{E}}$ pour le système $\mathrm{AgCl}-\mathrm{ZnCl}_{2}$ et pour le système $\mathrm{AgBr}-\mathrm{ZnBr}_{2}$ 
L'enthalpie libre molaire intégrale de mélange $\Delta \mathrm{G}^{\mathrm{M}}$ révèle les mêmes valeurs négatives pour les deux systèmes dans le domaine de composition de la fraction molaire du halogénure d'argent entre 0 et 0.2 . Les résultats provenant de mesures calorimétriques [9] et de mesures de force électromotrice [10] montrent que l'enthalpie molaire intégrale de mélange $\Delta \mathrm{H}^{\mathrm{M}}$ est positive pour les deux systèmes et, en plus, les variations de cette grandeur avec composition dans les mélanges $\mathrm{AgCl}-\mathrm{ZnCl}_{2}$ et $\mathrm{AgBr}-\mathrm{ZnBr}_{2}$ sont presque identiques. Ces faits suggèrent que l'entropie molaire intégrale de mélange $\Delta S^{M}$ est positive pour le domaine de composition de la fraction molaire du halogénure d'argent entre 0 et 0.2 . Il semble aussi que les valeurs $\Delta \mathrm{S}^{\mathrm{M}}$ relatives au système $\mathrm{AgCl}-\mathrm{ZnCl}_{2}$ soient plus hautes en comparaison avec celles relatives au système $\mathrm{AgBr}-\mathrm{ZnBr}_{2}$, étant donné que les températures concernées sont plus élevées pour les mélanges $\mathrm{AgBr}-\mathrm{ZnBr}_{2}$ que pour les mélanges $\mathrm{AgCl}-\mathrm{ZnCl}_{2}$ (la valeur de température de fusion de $\mathrm{ZnCl}_{2}$ et de $\mathrm{ZnBr}_{2}$ est respectivement $587.0 \mathrm{~K}$ et $675.4 \mathrm{~K}$ ). Ceci est compatible avec notre connaissance sur la structure des halogénures de zinc fondus. Or, après fusion $\mathrm{ZnCl}_{2}$ forme un liquide associé, construit avec unités tétraèdriques enchaînés mutuellement[11-14], la viscosité de $\mathrm{ZnCl}_{2}$ fondu étant dix fois plus grande que celle de $\mathrm{ZnBr}_{2}$ [15]. Il est donc possible que des changements de l'entropie lors de la formation du mélange soient plus importantes pour le système $\mathrm{AgCl}-\mathrm{ZnCl}_{2}$ par rapport à ceux de $\mathrm{AgBr}-\mathrm{ZnBr}_{2}$.

\section{Remerciements}

Ce travail a été soutenu par KBN, Pologne (Grant No 7 T09A 089 21)

\section{Références}

1. H. Hefang, M. Fuding, J. D. Mackenzie, J. Non-Cryst. Solids 55, 169 (1983).

2. L. F. Santos, R. M. Almeida, J. Non-Cryst. Solids 184, 68 (1995).

3. E. Kartini, M. F. Collins, F. Mezei, E. C. Svensson, Physica B 241-243, 909 (1998).

4. N. I. Glistenko, G. I. Artemova, Trudy Khim. Fak. Voronezh. Gos. Univ. 32, 87 (1953).

5. W. T. Thompson, S. N. Flengas, Can. J. Chem. 49, 1550 (1971).

6. O. Kubaschewski, C. B. Alcock, P. J. Spencer, Materials Thermochemistry, $6 \mathrm{e}$ édn. (Pergamon Press Ltd, England, Oxford 1993)

7. A. Winzer, Kristall und Technik 14, 51 (1979).

8. I. Bibas, J. Leonardi, Compt. Rend. Acad. Sci. Paris, Ser. C 268, 877 (1969).

9. G. N. Papatheodorou, O. J. Kleppa, Z. anorg. allg. Chem. 401, 132 (1973).

10. E. Kundys, Pol. J. Chem. 59, 487 (1985).

11. S. Biggin, J. E. Enderby, J. Phys. C: Solid State Phys. 14, 3129 (1981).

12. D. A. Allen, R. A. Howe, N. D. Wood, W. S. Howells, J. Chem. Phys. 94, 5071 (1991).

13. J. Neuefeind, K. Tödheide, A. Lemke, H. Bertagnolli, J. Non-Cryst. Solids 224, 205 (1998).

14. Y. Okamoto, K. Fukushima, Y. Iwadate, J. Non-Cryst. Solids 312-314, 450 (2002).

15. M. V. Susic, S. V. Mentus, J. Chem. Phys. 62, 744 (1975). 